\title{
Surging Prevalence of Cryptogenic Cirrhosis in Type-2 Diabetes Mellitus: An Alarming Fact or a Big Illusory Bias?
}

\section{Abbas $\mathrm{TA}^{1 *}$ and Razavian $\mathrm{NZ}^{2}$}

${ }^{1}$ Department of Internal Medicine, Shahrood Azad University Medical School, Iran ${ }^{2}$ Department of Hepatology, Shahrood Azad University Medical School, Iran

\begin{abstract}
Background: A growing body of evidence would consider the cryptogenic cirrhosis as the immediate offspring of Type 2 diabetes mellitus (T2DM). But, what we routinely observe here in Iran seems totally different. We would suggest that, the majority of reported cases of cryptogenic cirrhosis are actually the result of surreptitious or under-estimated alcohol consumption in a subject suffering from metabolic syndrome, not the direct consequence or as a blooming belief, a chronic complication of T2DM. In order to clarify the issue, a population of T2DM subjects living in a closed, religious society in which alcohol production, distribution, and consumption were legally and also ritually controlled, were investigated for manifestations of end-stage liver disease.
\end{abstract}

Materials and methods: In a prospective observational study, one hundred and thirty-two T2DM subjects (43 males and 89 females) were consecutively included. They were interviewed and carefully examined for any stigmata of chronic liver failure and cirrhosis. Then, a comprehensive set of liver function tests not to mention viral and immunological markers and also abdominal sonography were carried out. Liver biopsy was considered once academically indicated.

Results: The average age of patients was $58 \pm 10.9$ years (range, 31-83 years), and the duration of diabetes was $7.4 \pm 6$ years (range, $1-25$ years). BMI came out as $34.9 \pm 5.3$ with mean waist circumference of $71.2 \pm 11.5 \mathrm{~cm}$. To our surprise, from 132 T2DM patients only eight cases $(6 \%)$ displayed mild to moderate abnormal liver enzymes level, of which only 2 cases had liver enzymes more than twice normal. With regard to our main concern, only two subjects $(1.5 \%)$ manifested overt liver failure and cirrhosis, of whom, one person tested positive for HCVAb and the other disclosed eventually to have been drinking alcohol at younger ages for years while living abroad.

Conclusion: We would suggest, if alcoholic liver disease -as a major confounding variable- becomes efficiently eliminated from the relevant studies, T2DM, per se, would seldom persist as a direct causative risk for cryptogenic cirrhosis. We, as a matter of quite conceivable fact, believe that T2DM, NAFLD and cryptogenic cirrhosis are all the results of a superior pathogenic process, the metabolic syndrome or better to say insulin resistance syndrome.

Keywords: Type-2 diabetes mellitus; Alcoholic liver disease; Cryptogenic cirrhosis; Metabolic syndrome

\section{Introduction}

Cirrhosis is defined as the advanced stages of hepatic fibrosis with characteristic distortion of hepatic architecture in addition to numerous regenerative nodules. Cirrhosis without an apparent cause (e.g. alcoholic liver disease, viral hepatitis B and C, autoimmune hepatitis, Wilson's disease and so on) has been labelled as cryptogenic [1,2]. Since the identification of metabolic syndrome as a distinct medical entity and addressing the insulin resistance and compensatory hyperinsulinemia as the basic pathophysiologic process, most of cryptogenic cirrhosis cases are considered to be linked to non-alcoholic fatty liver disease (NAFLD) of insulin resistance and metabolic syndrome. In this context, T2DM was mistakenly considered as a component of galloping metabolic syndrome or something equivalent to it, and the medical journals were flooded with papers addressing the cryptogenic cirrhosis as the direct offspring of T2DM [3-9]. The under-estimated and usually under- reported alcohol consumption by general population on the one hand, and profound de novo impairment of glucose tolerance in chronic liver failure on the other, create a perplexing situation in which, one would not easily figure out which one is coming first; T2DM or cirrhosis! In a study conducted by Younossi, of 132 patients with NAFLD, 44 subjects (33\%) were reported to have established T2DM [10]. Of those forty- four diabetic patients with NAFLD, 11 were reported to be affected by frank cryptogenic cirrhosis. Going through the paper, you would immediately suppose that, one out of three type 2 diabetic patients have had concomitant end-stage cirrhosis of the liver. In a review article, Cusi proceeded further up and stated that approximately
$70 \%$ of $\mathrm{T} 2 \mathrm{DM}$ patients had fatty liver, and the non-alcoholic steatohepatitis was suggested as the leading cause of end- stage liver disease in persons with T2DM. The author eventually concluded that, NASH is a severe, straightforward "complication" of T2DM!! [11]. In Verona diabetes study from Paris (the mecca of finest wine in the world), de Marco made an statement that, end-stage hepatocellular failure due to cryptogenic cirrhosis was the fourth leading cause of death in type 2 diabetic population. In another case-control study carried out by Poonawala et al., of 49 patients defined as cryptogenic cirrhosis, $47 \%$ had T2DM. They easily concluded that one out of two cases of cirrhosis with undetermined etiology (cryptogenic) was affected by T2DM, so the two medical entities were supposed to be unequivocally linked with respect to their pathogenesis [12]. It seems as though these authors completely ignored the already-settled issue of glucose intolerance and mild diabetes in most cases of end-stage liver disease. Contrary to the mentioned studies, in a more reasonable cohort study carried out in Japanese workers, NAFLD was presented as a strong predictive

*Corresponding author: Abbas TA, Endocrinologist, Department of Interna Medicine, Shahrood Azad University Medical School, Iran, Tel: 982332392661 E-mail: dr.tavakolian@gmail.com

Received February 10, 2017; Accepted March 31, 2017; Published April 06, 2017

Citation: Abbas TA, Razavian NZ (2017) Surging Prevalence of Cryptogenic Cirrhosis in Type-2 Diabetes Mellitus: An alarming Fact or a Big Illusory Bias? J Neurol Disord 5: 337. doi:10.4172/2329-6895.1000337

Copyright: (c) 2017 Abbas TA, et al. This is an open-access article distributed under the terms of the Creative Commons Attribution License, which permits unrestricted use, distribution, and reproduction in any medium, provided the original author and source are credited. 
risk for T2DM [13]. We believe that, T2DM has, per se, nothing to do with NAFLD, cryptogenic cirrhosis or hepatocellular carcinoma. We believe that T2DM and cryptogenic cirrhosis are both the victims of a superior pathophysiologic process, the "insulin Resistance syndrome". We would suggest that, there are two major background variables which have been easily neglected; the pervasive and obviously difficult to avoid under- reported alcohol consumption and second, the newly emerged, worldwide pandemic insulin resistance syndrome. To shed a faint beam of light onto this upside- down situation, we designed and carried out this simple but conclusive study. We took advantage of unique opportunity to investigate the prevalence of decompensated cryptogenic cirrhosis in a population of type 2 diabetic patients living in a society in which alcohol production; distribution and consumption were religiously, ritually and legally prohibited.

\section{Materials and Methods}

In a prospective observational study, a total of 132 overt T2DM patients attending a university hospital diabetes clinic were comprehensively informed regarding the research project and those who were deeply interested to participate were consecutively included. As mentioned, the study was carried out in a community in which, alcohol production, distribution, vending and consumption were strictly banned. Nonetheless, we considered any given hint towards the possibility of surreptitious alcohol intake as an absolute exclusion criterion. Subjects on long- term drugs, known to cause liver damage or affect PT, PTT, INR or platelets were excluded from the study. An experienced diabetologist in concert with a hepatologist patiently interviewed and thoroughly examined the patients (Table 1). Age, sex, duration of diabetes, the way of diabetes management, currently used medications, relevant past histories, weight, height and waist circumference were all recorded. The hepatologist was requested to carefully go through a provided list of clinical stigmata of chronic liver disease and cirrhosis including spider angiomata, palmar erythema, finger clubbing, Dupuyetren's contracture, gynecomastia, hepatomegaly, splenomegaly, ascites, collateral veins with caput medusas, Jaundice, fetor hepaticus and asterixis. A panel of blood tests including CBC, diff, platelet count, ALT, AST, alkaline phosphatase, bilirubin, serum albumin, PT, PTT, INR, HBsAg and HCVAb were carried out. Abdominal sonography was applied as the preferred imaging technique. Liver biopsy was to be considered once diagnostically indicated. To predict cirrhosis clinically and biochemically, we used the Udell GA's meta - analysis findings based on the presence of ascites, platelet count, spider angiomata and Bonacini cirrhosis discrimination score. Bonacini score was calculated from platelets count, liver enzyme levels and INR values. Data were captured and analysed by SPSS version 19. Results were reported as mean \pm SD for quantitative variables and percentages for categorical variables.

\section{Results}

A total of one hundred and thirty-two patients with T2DM (43 males and 89 females) finished the whole process of cirrhosis evaluation. The average age of patients was $58 \pm 10.9$ years (range, 31 83 ), and the mean duration of diabetes was $7.4 \pm 6$ years (range, $1-25$

\begin{tabular}{|c|c|c|c|c|}
\hline Variables & Mean & SD & Minimum & Maximum \\
\hline Age (yrs) & 58 & 10.9 & 31 & 83 \\
\hline Duration of T2DM (yrs) & 7.6 & 6 & 1 & 25 \\
\hline BMI & 34 & 5.3 & 21.7 & 52.5 \\
\hline $\begin{array}{c}\text { Waist Circumference } \\
\text { (Cm) }\end{array}$ & 71 & 11.5 & 50 & 150 \\
\hline
\end{tabular}

Table 1: Baseline characteristics of diabetic participants.

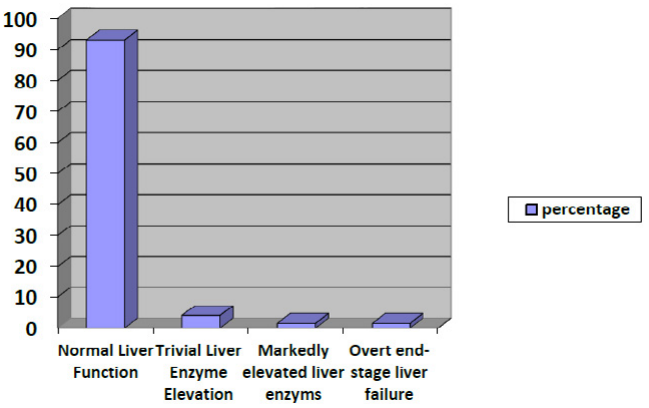

Figure 1: Percentage of T2DM patients with and without impaired liver function tests.

years) With regard to anthropometric measurements, mean of BMI was $34.9 \pm 5.3$ (range, $21.7-53.3$ ) and mean waist circumference was $71.2 \pm 11.5 \mathrm{~cm}$ (range, $50-150 \mathrm{~cm}$ ). On physical examination only two cases had hepato-splenomegaly, one case displayed mild Jaundice and two subjects were detected to have ascites. Quite surprising were the liver function test results. Of 132 diabetic individuals, only eight cases (6\%) showed abnormal liver enzyme levels, of which only two cases demonstrated ALT of more than twice normal. Markedly abnormal values for PT, INR and serum albumin were tracked in only two patients (1.5\%). According to Udell's meta-analysis cirrhosis criteria, 2 out of 132 diabetic subjects could be labelled as having concomitant decompensated cirrhosis, and six were supposed to be only affected by NASH. Of the two well-established cases of chronic liver failure, one (a 60-year-old aristocrat man) died of recurrent upper GI bleeding (Figure 1). After his death, his wife recalled, or better to say, confessed that her husband used to drink heavily for thirty years while living abroad. The second case of decompensated cirrhosis was an old lady who was tested positive for $\mathrm{HCV}$ antibody.

\section{Discussion}

A swelling body of evidence suggests a cause and effect relationship between T2DM and cryptogenic cirrhosis of the liver. Some authors claimed that, close to 50 percent of subjects affected byT2DM were suffering from concomitant NAFLD and almost one third had decompensated cirrhosis. Similarly, there are other reports expressing that, $70 \%$ of T2DM patients have fatty livers. Another paper openly stated that cryptogenic cirrhosis was the fourth leading cause of death in type 2 diabetic patients [10-12]. Whereas, our study indicated that, only six percent of T2DM subjects were affected by mild negligible NASH and less than $1.5 \%$ had concomitant cirrhosis or better to say overt decompensated cirrhosis, of which one patient tested positive for $\mathrm{HCV} \mathrm{Ab}$ and the other disclosed to be alcohol consumer for many years in the past. Despite the frank contradiction between our findings and some of the previously mentioned papers, one would be able to track the medical literature for some masterly designed and devotedly conducted studies which are in close agreement with our study. With the aim of examining the trues and tricks concerning the already reported increased risk of NAFLD and hepatocellular carcinoma in T2DM, El-seragetal., carried out a meaningful case-control study through which they concluded that, diabetes mellitus does increase the risk of hepatocellular carcinoma, but only in the presence of other risk factors such as hepatitis B or C or alcoholic cirrhosis [14]. This was exactly what we were trying to prove. We believe that T2DM has, per se, nothing to do with NASH, cryptogenic cirrhosis or hepatocellular carcinoma. As a matter of fact, T2DM, liver steatosis, NASH and cryptogenic cirrhosis are all victims of a prime pathogenic culprit, the devastating moderntime human health catastrophe, the insulin resistance syndrome. 
A must-to-know issue is the true location of T2DM in the Jigsaw of metabolic syndrome, a matter which has been the main source of all partialities and errors in relevant studies. As a component of active metabolic syndrome, NAFLD and NASH need severe, persistent and protracted hyperinsulinemia for vivid development and progression towards the cryptogenic cirrhosis. Right in contrast to galloping NASH, a state of relative insulin deficiency is needed to induce impairment of fasting plasma glucose (IFG) and then overt T2DM. In other words, for development of T2DM the process of metabolic syndrome and hyperinsulinemia must be loosened up or becomes somehow aborted. Therefore, from pathophysiologic point of view, T2DM and progressive NASH are situated on the opposite directions. According to the results of our study and the previously discussed pathophysiologic principles, we would reasonably suggest that, if the alcoholic liver disease, as the major and easily overlooked confounding variable, becomes efficiently eliminated from the studies, T2DM per se, would seldom cause a deepseated cirrhosis and fully-developed, end-stage liver failure. We, in fact, consider the rapidly growing incidence and the galloping prevalence of cryptogenic cirrhosis as the cumulative impacts of alcoholic liver disease and the damaging effects of unleashed hyperinsulinemia of insulin resistance syndrome, and in- between the T2DM is an innocent by-stander despite of being frequently seen along with cryptogenic cirrhosis. The supposition of any pathogenic link between T2DM and NAFLD is basically erroneous. Along the evolutional path of metabolic syndrome, from birth to death, NAFLD is situated on the first half of this path, whereas T2DM gradually appears over the second.

\section{Conclusion}

NAFLD develops and progresses on account of conspicuous hyperinsulinemia, while the impaired fasting glucose and overt T2DM occur once the pancreatic B- cells become exhausted and the excessive insulin secretion begins to fade away. Therefore, NAFLD and T2DM are, indeed, the two opposite aspects of a single pathophysiologic coin, that is, the insulin resistance syndrome. We would suggest that, in recent studies addressing the interrelationship between T2DM and NAFLD, two major background variables are being neglected; the pervasive and obviously difficult to prove under- reported alcohol consumption and the newly emerged, worldwide insulin resistance syndrome. In effect, the horrendous prevalence of NAFLD and cryptogenic cirrhosis is the summation effects of surreptitious alcoholic liver disease and the hyper-insulinemia phase of metabolic syndrome, whereas, T2DM is an innocent by-stander playing probably no significant role in the pathogenesis of non-alcoholic fatty liver disease and cryptogenic cirrhosis.

\section{References}

1. Ludwig J, Viggiano TR, McGill DB, Oh BJ (1980) Non-alcoholic steatohepatitis: Mayoclinic experiences with a hitherto unnamed disease. Mayo Clinic Proc 55 434-438.

2. Caldwell SH, Oelsner DH, lezzoni JC, Hespenheide EE, Battle EH, et al. (1999) Cryptogenic cirrhosis: Clinical characterization and risk factors for underlying disease. Hepatology 29: 664-669.

3. Trombetta M, Spiazzi G, Zoppini G, Muggeo M (2005) Type 2 diabetes and chronic liver disease in the Verona diabetes study. Aliment pharmacol 22: 24-27.

4. Balkau $B$, Eschwège $E$, Ducimetière $P$, Richard JL, Warnet JM (1991) The high risk of death by alcoholic related disease in subjects diagnosed as diabetic and impaired glucose tolerance: The Paris prospective study after 15 years follow up. J Clin Epidemiol 44(6): 465-474.

5. Belcher G, Schernthaner G (2005) Changes in liver tests during 1-year treatment of patients with T2DM with Pioglitazon, Metformin or Gliclizide. Diabet med 22: 973-979.

6. Lebovitz HE, Kreider M, Freed MI (2002) Evaluation of liver function in T2DM during clinical trials: evidence that Rosiglitazone does not cause hepatic dysfunction. Diabetes care 25: 815-821.

7. De Marco R, Locatelli F, Zoppini G, Verlato G, Bonora E, et al. (1999) Cause specific mortality in type 2 diabetes: The Verona diabetes study. Diabetes Care 22: 756-761.

8. Tolman GK, Fonseca V, Dalpiaz A, Tan MH (2007) Spectrum of liver disease in T2DM and management of patients with diabetes and liver disease. Diabetes care 30: 734-743.

9. Silverman JF, O'Brien KF, Long S, Leggett N, Khazanie PG, et al. (1990) Liver pathology in morbidly obese patients with or without diabetes .Am J Gastroentrol 85: 1349-1355.

10. Younossi ZM, Gramlich T, Matteoni CA, Boparai N, Mc Cullough AJ (2004) Nonalcoholic liver fatty disease in patients with type 2 diabetes. Clin Gastroentrol Hepato 2(3): 262-265.

11. Cusi K (2009) Non-alcoholic fatty liver disease in type 2 diabetes mellitus. Curr Opin Endoc Diabetes Obes 16(2): 141-149.

12. Poonawala A, Nair SP, Thuluvath PJ (2007) Prevalence of obesity and diabetes in patients with cryptogenic cirrhosis: A case - control study. Hepatology 32 689-692.

13. Shibata M, Kihara Y, Taguchi M, Tashiro M, Otsuki M (2007) Non-alcoholic fatty liver disease is a risk factor for Type 2 diabetes in middle - aged Japanese men. Diabetes Care 30: 2940-2944.

14. El-Serag HB, Richardson PA, Everhart JE (2001) The role of diabetes in hepatocellular carcinoma: A case-control study among USA veterans. Am J Gastroentrol 96(8): 2462-2467. 